\title{
Endoscopic ultrasound-guided fine-needle biopsy in patients with unexplained diffuse gastrointestinal wall thickening
}

\section{(ㄷ)(이우}

Authors

Carlos Chavarría ${ }^{1}$, Francisco J. García-Alonso ${ }^{1}$, Marina de Benito-Sanz ${ }^{1}$, Pilar Mata-Romero', Beatriz Madrigal²,

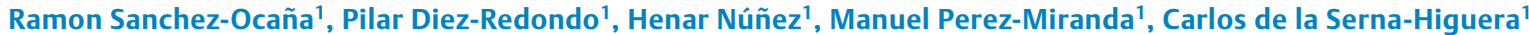

Institutions

1 Endoscopy Department, Hospital Universitario Río Hortega, Valladolid, Spain

2 Pathology Department, Hospital Universitario Río Hortega, Valladolid. Spain

submitted 19.5.2020

accepted after revision 7.8 .2020

Bibliography

Endosc Int Open 2021; 09: E1466-E1471

DOI 10.1055/a-1526-0407

ISSN 2364-3722

(c) 2021. The Author(s).

This is an open access article published by Thieme under the terms of the Creative Commons Attribution-NonDerivative-NonCommercial License, permitting copying and reproduction so long as the original work is given appropriate credit. Contents may not be used for commercial purposes, or adapted, remixed, transformed or built upon. (https://creativecommons.org/licenses/by-nc-nd/4.0/)

Georg Thieme Verlag KG, Rüdigerstraße 14,

70469 Stuttgart, Germany

Corresponding autor

Carlos Chavarría, MD, PhD, Gastroenterology Unit, Hospital

Universitario Río Hortega , Dulzaina, 2, 47012 Valladolid,

Spain

Fax: +34-98320400

chavarria.carlosm@gmail.com

\section{ABSTRACT}

Background and study aims Endoscopic ultrasoundguided fine needle biopsy (EUS-FNB) is recommended after non-diagnostic biopsy in gastrointestinal wall thickening, although the performance of currently available FNB needles in this setting is unknown. We aimed to assess the diagnostic accuracy and safety of EUS-FNB and to evaluate the "T" wall staging in malignant pathology.

Patients and methods This was a single center retrospective study that included all consecutive patients undergoing EUS-FNB for diffuse gastrointestinal wall thickening with at least one previous negative conventional endoscopic biopsy between January 2016 and November 2019. EUS-FNB was performed using linear-array echoendoscopes with slowpull/fanning technique. Tissue acquisition was done with 19- or 22-gauge biopsy needles. Samples were included in formalin without rapid on-site evaluation and submitted for histopathological processing. The final diagnosis was based on conclusive histology or absence of evidence of disease progression after follow-up at least 6 months.

Results Twenty-nine patients (21 men), with a median age of 68 (IQR: 56-77), were included. EUS-FNB was technically feasible and the sample quality was adequate for full histological assessment in all patients (100\%). Sensitivity, specificity, positive and negative predictive values, and overall accuracy for diagnosis of malignancy were $95.5 \%, 100 \%$, $100 \%, 83.3 \%$, and $96.3 \%$, respectively. In patients with malignant disease, the samples obtained allowed detection of signs of deep layer infiltration ("histological staging") in 17 of 21 cases (81\%). No adverse events were noted.

Conclusions The EUS-FNB technique demonstrated excellent diagnostic performance and safety in the study of unexplained diffuse gastrointestinal wall thickening. Histological staging was obtained in a high percentage of samples.

\section{Introduction}

Diffuse gastrointestinal wall thickening is predominantly observed in the stomach and, less frequently, in the esophagus and rectum. The differential diagnosis is broad. Malignant causes include adenocarcinoma, plastic linitis and, less frequently, lymphoma or metastasis. Benign conditions are multiple and include infiltrative disorders and infectious diseases, among others [1,2]. Therefore, accurate diagnosis is essential for prognosis and treatment.

The diagnostic process is difficult and usually requires a combination of multiple tests, including abdominal computed tomography (CT) and endoscopic tissue sampling. However, conventional biopsies can be falsely negative, especially in submucosal infiltrating tumors [3]. To increase the diagnostic yield, various techniques have been suggested, such as the 
"bite on bite" biopsy technique with jumbo forceps, mucosal resection and submucosal dissection techniques [4-7]. However, the improved diagnostic profitability is frequently achieved at the expense of an increased risk of bleeding and perforation.

Endoscopic ultrasound (EUS) is the diagnostic procedure of choice to study subepithelial lesions and delineate the gastrointestinal wall layers. Although there are endosonographic criteria to differentiate malignancy from benignity, a definitive diagnosis requires a histological sample [4], which can be obtained in the same procedure through EUS-guided fine-needle aspiration (FNA) [5]. Unfortunately, EUS-FNA has shown suboptimal results in diffuse gastrointestinal wall thickening [6]. Moreover, the assessment of the architectural pattern is limited, which is an important drawback in conditions such as plastic linitis, lymphomas and gastrointestinal stromal tumors. To overcome these issues, core biopsy needles have been developed. The ability of fine needle biopsy (FNB) to obtain deeper or even full-thickness "histological" wall samples may improve the diagnostic yield in detecting underlying malignancy [7, 8], but the available published evidence is still scarce.

Thus, we aimed to assess the diagnostic accuracy and safety of EUS-FNB. As a secondary objective, we reviewed whether the samples obtained allowed "T" wall staging in malignant pathology.

\section{Patients and methods}

This was a single-center, retrospective case series assessing the diagnostic performance of EUS-FNB. The study was approved by the institutional review board. All patients provided written informed consent for the procedure and were aware of the use of histological puncture needles.

\section{Study population}

We included all consecutive patients who underwent EUS-FNB at a large tertiary referral center for confirmed diffuse gastrointestinal wall thickening with at least one previous negative conventional endoscopic biopsy between January 2016 and November 2019. Gastrointestinal wall thickening was confirmed either with a CT scan or during the EUS. Eligible patients were retrieved from a prospective database kept in our unit which includes all consecutive patients undergoing EUS in our center. Variables included in this database thoroughly summarize the procedure.

Data from electronic medical records were abstracted and included demographics, clinical and radiological exams, previous endoscopic procedures, and adverse events (AEs). Follow-up was defined as the interval from EUS-FNB to the last available outpatient visit or death. Follow-up visits were performed at the discretion of the responsible physician.

\section{EUS-FNB sampling protocol}

EUS-FNB was performed using linear-array echoendoscopes (Olympus GF-UCT 140) by a single expert operator (C.S.H). Tissue acquisition was done with a 19- or 22-gauge biopsy needle EchoTip ProCore (Cook Medical), AcquireTM (Boston Scientific) or SharkcoreTM (Medtronic). A radial echoendoscope (Olympus GF-UM 160) was previously used to examine the gastrointestinal wall layers and to identify the section of the wall with the maximum thickness ( $\mathbf{F i g}$. 1a). After the target area was endosonographically visualized and the region scanned for vessels using color and pulsed Doppler, the biopsy was performed either from the esophagus, stomach, duodenum or rectum depending on the location of the lesion. With the stylet retracted but still inside the needle, the biopsy needle was moved forward into the lesion under full real-time ultrasound control. Once the lesion had been penetrated, the stylet was removed slowly and suction was applied for 10 to 20 seconds using a 20-mL syringe while performing a slow-pull and/or fanning technique by moving the needle to and fro 10 to 15 times obliquely through the wall layers under EUS guidance avoiding penetration of serosa ( $\triangleright$ Fig. 1b). Suction was released before removing the needle. Three to five needle passes were performed at the discretion of the operator. There was no pathologist in the endoscopy room and tissue samples were recovered in a liquid-based preparation, (CytorichRed) by flushing the needle with $5 \mathrm{cc}$ of saline. All samples were processed to provide cell blocks for histological analysis. All the tissue cores obtained were examined by a single histopathologist (B.M.R) with over 15 years of expertise in evaluating tissue materials obtained via EUS. Samples were embedded in paraffin. Tissue sections of 3 to $4 \mu \mathrm{m}$ were stained by the hematoxylin-eosin technique for morphological evaluation and/or different immunohistochemical analysis (> Fig.2).

\section{Study endpoints}

The primary outcome of interest was the diagnostic accuracy of EUS-FNB. To estimate the diagnostic accuracy, diagnoses of malignancy were based on operative histology if available. In patients not undergoing surgery, the EUS-FNB malignancy diagnoses were considered definitive if the patient died from malignant disease, showed disease progression during follow-up or presented imaging findings suggesting advanced malignant disease and received radio/chemotherapy. For patients with benign lesions or non-pathologic findings, the diagnosis required confirmation either with the histopathological examination of the surgically resected specimen or with a follow-up $\geq 6$ months without changes in imaging and/or endoscopic evaluation.

Secondary outcomes of interest were to the rate of AEs and their severity according to the American Society for Gastrointestinal Endoscopy (ASGE) lexicon [9] and, to assess the contribution of EUS-FNB to the "T" wall staging in malignant pathology. For this purpose, we considered a sample as "adequate for histological assessment" when an architecturally intact piece of tissue was stated as sufficient for histological evaluation in the pathological report by the histopathologist (B.M.R). Layers 1 (superficial mucosa) and 2 (deep mucosa) were considered superficial, while the remaining layers, 3 (submucosa and interface), 4 (muscularis propria) and 5 (serosa) were considered deep [10]. Micro-ascites was defined as a minimal amount of intraperitoneal free liquid detected during EUS not noticed in previous abdominal imaging. 

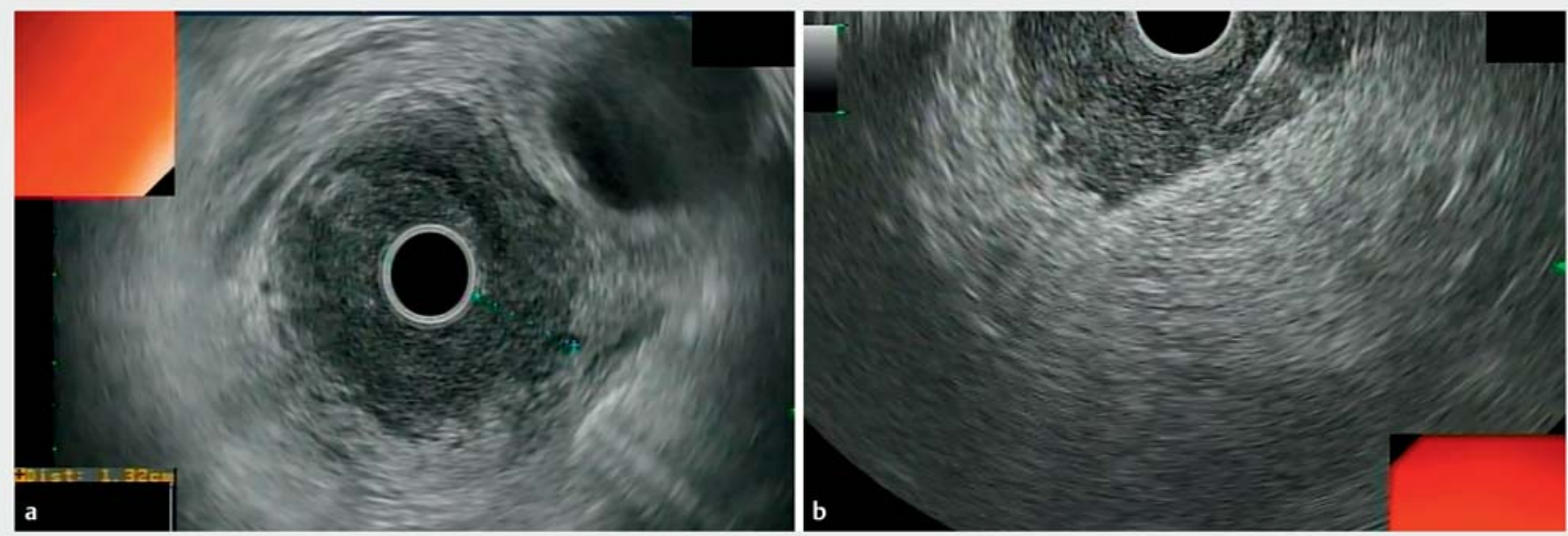

Fig. 1 EUS-guided fine needle biopsy of the gastric wall. a Radial EUS showing thickened gastric wall with indistinct wall layers. b Puncture by using a curvilinear echoendoscope and the tissue tray advanced obliquely but not penetrating through serosa.
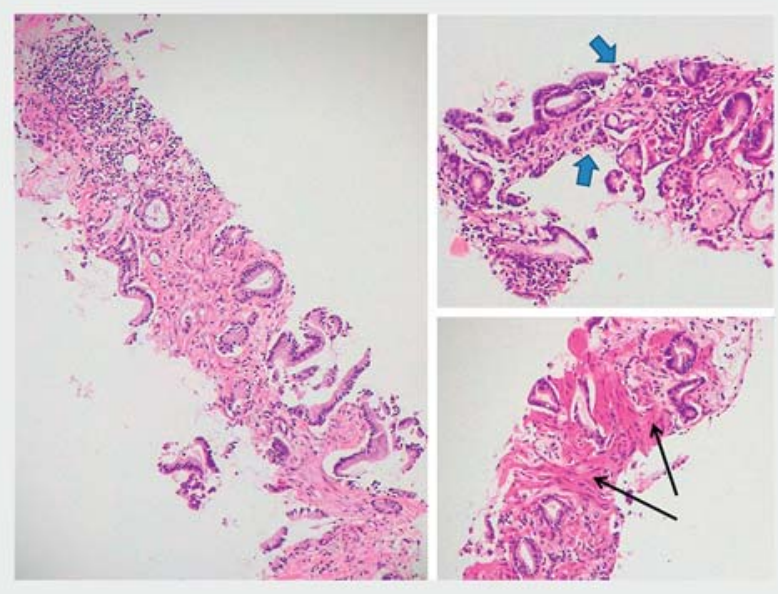

Fig. 2 Gastric adenocarcinoma. Histology (H\&E 10x) of transmural gastric wall specimen showing deep neoplastic infiltration. The sample includes mucosa (blue arrows), submucosa and muscularis propria (black arrows), infiltrated by a proliferation of neoplastic glands of different sizes, surrounded by desmoplastic stroma.

\section{Statistical analysis}

The statistical analysis was carried out using Stata (StataCorp. 2013. College Station, Texas, United States). Continuous variables were summarized using mean and standard deviation (SD) or median and interquartile range (IQR) in case they did not present a normal distribution according to the Kolmogorov-Smirnov test; categorical variables were expressed as percentages. The Pearson's $\mathrm{X} 2$ test was used to assess differences between categorical values (in case the expected frequencies in contingency tables were lower than 5, the Fisher's exact was performed). The Student's t-test or Wilcoxon's test were used to assess association between continuous and categorical variables. A level of significance of $P<.05$ was adopted for all inferential testing.

\section{Results}

A total of 29 patients with a median age of 68 (IQR: $56-77$ ) years, 21 males (72.4\%), were included. Patient baseline characteristics and EUS findings are summarized in $>$ Table 1 . The median number of endoscopic procedures with biopsies before EUS-FNB was two (1-3). The histological diagnoses of the conventional endoscopic biopsies were chronic inflammation in 22 patients (75.5\%) and normal mucosa in seven patients (24.5\%).

On EUS examination, the median wall thickness according to the location of the lesion was: esophagus 9.7 mm (IQR: 3-15), stomach $10.9 \mathrm{~mm}$ (IQR: 5-30), duodenum $7.5 \mathrm{~mm}$ (IQR: 5-10), rectum $7.0 \mathrm{~mm}$ (IQR: 5-10) and the only case of esophago-jejunal anastomosis was $2 \mathrm{~mm}$. Most patients, 21 (72.4\%), presented a loss of the transmural layer pattern.

Locoregional lymphadenopathies were observed in six patients (21\%) and ascites in 12 cases (52.1\%), five of them micro-ascites. Locoregional lymphadenopathies were observed in $28.6 \%$ of patients with malignancy and were absent in the benign findings $(P=0.18)$. Ascites was identified in $66.7 \%(12 / 18)$ of the malignant cases and in none of the benign ones $(P=$ $0.01)$. The layer pattern was preserved in $66.7 \%(4 / 6)$ of the benign lesions, but only in $9.5 \%$ of the malignant ones $(P=0.01)$.

The Procore $19 \mathrm{G}$ needle was used in only two cases (7\%) cases, while the Acquire 22G and Sharkcore 22G needles in 14 patients (48\%) and 13 patients (45\%), respectively. EUS-guided biopsy was technically feasible in all 29 cases (100\%). The obtained samples were adequate for histological assessment in all patients (100\%). No AEs were reported. Malignancy was confirmed in 21 of 29 cases (72.4\%). The final histologic diagnosis is shown in - Table 2 . The diagnosis was made by histology after the EUS-FNB in 20 patients while one patient, a 70-yearold man whose final diagnosis was gastrointestinal lymphoma, resulted in a false negative. The final diagnosis was reached through a flow cytometry analysis of the second conventional endoscopy mucosal biopsies. EUS was performed before these results were available. 
- Table 1 Baseline characteristics of the patients and EUS findings.

\begin{tabular}{|l|c|}
\hline Patients & $\mathbf{n}=\mathbf{2 9}$ \\
\hline Male, $\mathrm{n}(\%)$ & $21(72.4)$ \\
\hline Median age, years (interquartile range) & $68(56-77)$ \\
\hline Anatomical location of the wall thickening, $\mathrm{n}(\%)$ & \\
\hline - Esophagus & $6(20.7)$ \\
\hline - Stomach & $15(51.7)$ \\
\hline - Duodenum & $4(13.8)$ \\
\hline - Rectum & $3(3.3)$ \\
\hline Esophago-jejunal anastomosis & $1(3.4)$ \\
\hline Presence of locoregional lymphadenopathies, $\mathrm{n}(\%)$ & $6(21)$ \\
\hline Presence of ascites, $\mathrm{n}(\%)$ & $12(52.1)$ \\
\hline Median number of passes, (IQR) & $4(3-5)$ \\
\hline Puncture needles, $\mathrm{n}(\%)$ & $2(7)$ \\
\hline - Procore 19 & $14(48)$ \\
\hline - Acquire 22G & $13(45)$ \\
\hline - Sharkcore 22 G & \\
\hline EUS, endoscopic ultrasound; IQR, interquartile range. & \\
\hline
\end{tabular}

Among the eight patients with benign diagnoses, two were lost to follow-up immediately after the procedure and six maintained the diagnosis unchanged after a median follow-up of 18 months (10-55). Thus, the diagnostic accuracy was assessed in 27 patients. We observed a $95.5 \%$ sensitivity $(95 \% \mathrm{Cl}: 77.2 \%-$ 99.9\%), $100 \%$ specificity ( $95 \% \mathrm{Cl}: 47.8 \%-100 \%$ ), $100 \%$ positive predictive value (95\% $\mathrm{Cl}: 83.9 \%-100 \%)$, and $83.3 \%$ negative predictive value (95\% Cl: 35.9\%-99.6\%). The overall diagnostic accuracy was $96.3 \%$ (95\%Cl: $81 \%-99.9 \%)$. In patients with nonlymphoid malignant disease, the samples obtained by EUS-FNB allowed detection of histological signs of deep layer infiltration ("histological staging") in 17 of 21 cases (81\%) $(95 \% \mathrm{Cl}$ : $60 \%-$ $92.3 \%$ ). Specifically, histological examination was able to assess the layered mural pattern and specify the depth of infiltration in 19 patients: T1: two cases, T2: 14 cases; T3: one case; and T4: two cases.

\section{Discussion}

Our study presents a single-center, retrospective case series assessing the role of EUS-FNB in diffuse gastrointestinal wall thickening. We observed an overall diagnostic accuracy of 96.3\% (95\%Cl: $81 \%-99.9 \%)$, with a $95.5 \%$ sensitivity $(95 \% \mathrm{Cl}$ : $77.2 \%-99.9 \%$ ) and $100 \%$ specificity (95\%Cl: $47.8 \%-100 \%)$, without any AEs.

Unexplained diffuse gastrointestinal wall thickening diagnosis remains a challenge in clinical practice. The European Society of Gastrointestinal Endoscopy (ESGE) guidelines suggest EUS-guided sampling after failed standard biopsy techniques, preferring core biopsy FNB over FNA [5], due to the poor results of FNA in this setting. The largest available study assessing EUSFNA included 103 gastrointestinal wall lesions (no stromal tumors), reporting a $61 \%$ sensitivity and a $67 \%$ accuracy [11]. A smaller case series reported 60 gastrointestinal wall lesions, with an $89 \%$ accuracy and $89 \%$ sensitivity, but nearly one-third of the sample were $\mathrm{Gl}$ stromal tumors, which probably acted as a confounding factor [12]. Another large case series with over 250 cases comparing the diagnostic yield of FNA in different locations, observed intramural lesions had the poorest results, with an $83 \%$ accuracy and $67 \%$ sensitivity [13]. A recently pub-

- Table 2 Details of EUS examination and final histological diagnosis.

\begin{tabular}{|c|c|c|c|}
\hline Location (n) & Length of tissue core $\mathrm{mm}$ (IQR) & \multicolumn{2}{|c|}{ Final histology (n) } \\
\hline \multirow[t]{2}{*}{ Esophagus (6) } & \multirow[t]{2}{*}{$10(5-15)$} & Malignant & $\begin{array}{l}\text { Adenocarcinoma (4) } \\
\text { Pulmonary adenocarcinoma (1) }\end{array}$ \\
\hline & & Benign & Leiomyoma (1) \\
\hline \multirow[t]{2}{*}{ Stomach (15) } & \multirow[t]{2}{*}{$10(5-10)$} & Malignant & $\begin{array}{l}\text { Adenocarcinoma ( } 8 \text { ) } \\
\text { Adenocarcinoma with signet ring cells ( } 3 \text { ) } \\
\text { Lymphoma (1) }\end{array}$ \\
\hline & & Benign & Chronic gastritis (3) \\
\hline \multirow[t]{2}{*}{ Duodenum (4) } & \multirow[t]{2}{*}{$7.5(5-10)$} & Malignant & $\begin{array}{l}\text { Adenocarcinoma (2) } \\
\text { Neuroendocrine tumor (1) }\end{array}$ \\
\hline & & Benign & Chronic duodenitis (1) \\
\hline Esophago-jejunal anastomosis (1) & 2 & Benign & Chronic inflammation (1) \\
\hline \multirow[t]{2}{*}{ Rectum (3) } & \multirow[t]{2}{*}{$10(5-15)$} & Malignant & Adenocarcinoma (1) \\
\hline & & Benign & $\begin{array}{l}\text { Indeterminate proctitis (1) } \\
\text { Normal (1) }\end{array}$ \\
\hline
\end{tabular}

EUS, endoscopic ultrasound; IQR, interquartile range. 
lished single-center smaller case series, including 18 EUS-FNA of thickened gastric walls, reported an $83.3 \%$ accuracy[14].

The available evidence supporting the preference for core biopsies, while scarce, presents better results. The largest available study is a retrospective case series including 31 cases of gastroesophageal wall thickening where samples were obtained with a EUS Tru-cut needle, achieving a diagnostic accuracy of $90 \%$ [6], while a smaller series of 10 cases also reached a $90 \%$ diagnostic accuracy [10]. Despite these results, Tru-cut needles have been abandoned due to their technical limitations, which caused frequent malfunctions. To overcome this, core biopsy needles have been developed $[6,15]$. Two large series with over 100 patients each reported adequate samples in $87.5 \%$ [16] and $97.5 \%$ [17] of procedures, but they only included two and 11 patients with gastrointestinal wall thickening, respectively.

In our study, the adequacy of the sample and the diagnostic yield of EUS-FNB improve the results achieved with FNA and are at least comparable to the smaller previous series of EUS-FNB. These outcomes can be due to multiple factors: 1) histological needle design facilitates transmural puncture. When performing the transmural puncture, the beveled profile of the needle tip could prevent the tenting effect seen with standard needles; 2 ) the new models of histological needles have improved the design of the first histological needles (Tru-cut needle), which were difficult to handle and associated multiple technical difficulties, thus reducing the number of unsuccessful procedures; and 3) an expert endoscopist and a dedicated pathologist participated in all procedures, which has been decisive in obtaining better results.

In this study, tissue core biopsies obtained by EUS-FNB transmural contributed to " $T$ " staging in most patients with nonlymphoid malignant pathology ( $81 \%)$. This finding is explained by the layered pattern with preserved architecture in the sample. These results need further evaluation, as, to our knowledge, there are no available studies comparing EUS-FNB T staging to post-surgical staging. If an accurate prediction of the depth of tumor penetration was achieved, it would be a valuable tool to plan therapeutic strategies (i.e., neoadjuvant chemotherapy).

Although the presence of ascites is associated with advanced tumor staging, the interpretation of the presence of micro-ascites in the staging of gastric cancer is controversial [18]. In our series, the five patients who had micro-ascites had malignant disease, reaching the muscular layer in two cases and the serous layer in the remaining three. Thus, we believe that our results support the presence of micro-ascites in EUS as an imaging criterion for deep transmural involvement. However, these findings are not backed by a robust gold standard such as an evaluation of the surgical specimen. Thus, further studies are needed to confirm the diagnostic accuracy of micro-ascites for deep infiltration.

Safety of EUS-FNA is well established, with AE rates ranging between $1 \%$ and $2.5 \%$ [19]. Different studies have found that the safety profile of EUS-FNB is similar to EUS-FNA $[8,16]$. In our series there were no complications associated with the pro- cedure, supporting the excellent safety profile of the procedure.

Our study has several limitations. First, only two patients in our cohort underwent surgery. Thus, the gold standard used to assess the diagnostic accuracy was a surrogate and the local staging could not be ascertained. Second, the retrospective design is prone to selection bias. EUS-FNB was probably performed only in patients with a high pre-test probability of malignancy. Therefore, the reported predictive values should be taken with caution. Third, as a single-center study, the results are very operator dependent. Both the endosonographer and the pathologist presented more than 15 years of experience in EUS and gastrointestinal pathology, respectively. Despite these limitations, this is the largest series published to date assessing the diagnostic accuracy of EUS-FNB using biopsy needles in the specific setting of gastrointestinal wall thickening.

\section{Conclusions}

In conclusion, our study suggests that EUS-FNB can attain an excellent diagnostic performance in the study of unexplained diffuse gastrointestinal wall thickening with an optimal safety profile. Histological staging was possible in a high proportion of patients, although its results could not be confirmed. Thus, our results support the recommendation of considering EUSFNB in patients with unexplained gastrointestinal wall thickening with non-diagnostic conventional mucosal biopsies and any abnormal EUS, laboratory or radiologic finding suggesting malignant disease.

\section{Competing interests}

Dr. Manuel Perez-Miranda receives consultancy and speaking fees from Boston Scientific, Olympus and M.I.Tech. The remaining authors declare that they have no conflict of interest.

\section{References}

[1] Sharma V, Rana SS, Bhasin DK. Diffuse gastric wall thickening: appearances can be deceptive. Clin Gastroenterol Hepatol 2015; 13: e121-122

[2] Kawano H, Ishii A, Kimura T et al. IgG4-related disease manifesting the gastric wall thickening. Pathol Int 2016; 66: 23-28

[3] Kwack WG, Ho W], Kim JH et al. Understanding the diagnostic yield of current endoscopic biopsy for gastric neoplasm: A prospective singlecenter analysis based on tumor characteristics stratified by biopsy number and site. Medicine (Baltimore) 2016; 95: e4196

[4] Lim H, Lee GH, Na HK et al. Use of endoscopic ultrasound to evaluate large gastric folds: features predictive of malignancy. Ultrasound Med Biol 2015; 41: 2614-2620

[5] Dumonceau JM, Deprez PH, Jenssen C et al. Indications, results, and clinical impact of endoscopic ultrasound (EUS)-guided sampling in gastroenterology: European Society of Gastrointestinal Endoscopy (ESGE) Clinical Guideline - Updated January 2017. Endoscopy 2017; 49: $695-714$ 
[6] Aithal GP, Anagnostopoulos GK, Kaye P. EUS-guided Trucut mural biopsies in the investigation of unexplained thickening of the esophagogastric wall. Gastrointest Endosc 2005; 62: 624-629

[7] Muraoka S, Tsuchida K, Iwasaki M et al. A case report of gastric linitis plastica diagnosed by endoscopic ultrasound-guided fine needle aspiration. Medicine (Baltimore) 2017; 96: e8937

[8] Iglesias-Garcia J, Abdulkader I, Larino-Noia J et al. Evaluation of the adequacy and diagnostic accuracy of the histology samples obtained with a newly designed 19-gauge EUS histology needle. Rev Esp Enferm Dig 2014; 106: 6-14

[9] Cotton PB, Eisen GM, Aabakken L et al. A lexicon for endoscopic adverse events: report of an ASGE workshop. Gastrointest Endosc 2010; 71: 446-454

[10] Gines A, Pellise M, Fernandez-Esparrach G et al. Endoscopic ultrasonography in patients with large gastric folds at endoscopy and biopsies negative for malignancy: predictors of malignant disease and clinical impact. Am J Gastroenterol 2006; 101: 64-69

[11] Wiersema M], Vilmann P, Giovannini M et al. Endosonography-guided fine-needle aspiration biopsy: diagnostic accuracy and complication assessment. Gastroenterology 1997; 112: 1087-1095

[12] Vander Noot MR 3rd., Eloubeidi MA, Chen VK et al. Diagnosis of gastrointestinal tract lesions by endoscopic ultrasound-guided fine-needle aspiration biopsy. Cancer 2004; 102: 157-163
[13] Pellise Urquiza M, Fernandez-Esparrach G, Sole M et al. Endoscopic ultrasound-guided fine needle aspiration: predictive factors of accurate diagnosis and cost-minimization analysis of on-site pathologist. Gastroenterol Hepatol 2007; 30: 319-324

[14] Tellez-Avila FI, Duarte-Medrano G, Lopez-Arce G et al. EUS-guided tissue samples for the diagnosis of patients with a thickened gastric wall and prior negative endoscopic biopsies. Acta Gastroenterol Belg 2019; 82: 359-362

[15] Yu L, Chen K, Xu Y et al. The value of EUS in combination with cytological, flow cytometry, and gene rearrangement in the diagnosis of gastrointestinal lymphoma. Hematol Oncol 2017; 35: 303-309

[16] Iglesias-Garcia J, Poley JW, Larghi A et al. Feasibility and yield of a new EUS histology needle: results from a multicenter, pooled, cohort study. Gastrointest Endosc 2011; 73: 1189-1196

[17] Larghi A, Verna EC, Ricci R et al. EUS-guided fine-needle tissue acquisition by using a 19-gauge needle in a selected patient population: a prospective study. Gastrointest Endosc 2011; 74: 504-510

[18] Lee YT, Ng EK, Hung LC et al. Accuracy of endoscopic ultrasonography in diagnosing ascites and predicting peritoneal metastases in gastric cancer patients. Gut 2005; 54: 1541-1545

[19] Early DS, Acosta RD. ASGE Standards of Practice Committee. et al. Adverse events associated with EUS and EUS with FNA. Gastrointest Endosc 2013; 77: 839-843 